\title{
Tuberculosis in pregnancy
}

Lucian Gheorghe Pop ${ }^{1 *}$, Nicolae Bacalbasa ${ }^{2,3}$, Ioan Dumitru Suciu ${ }^{4}$, Paris Ionescu ${ }^{5}$, Oana Daniela Toader ${ }^{1,2}$

\section{Author Affiliations:}

1. Department of Obstetrics and Gynecology, Alessandrescu-Rusescu National Institute for Mother and Child Health, Bucharest, Romania

2. Department of Obstetrics and Gynecology, Carol Davila University of Medicine and Pharmacy, Bucharest, Romania

3. Center of Excellence in Translational Medicine, Fundeni Clinical Institute, Bucharest, Romania

4. Department of General Surgery, Clinical Emergency Hospital of Bucharest, Bucharest, Romania

5. Department of Obstetrics and Gynecology, Ovidius University, Constanta, Romania

\author{
* Corresponding Author: \\ Lucian Pop, MD, \\ Department of Obstetrics and \\ Gynecology, \\ Alessandrescu-Rusescu National \\ Institute of Mother and Child \\ Health, Bucharest, Romania. \\ Phone: +40741044720 \\ E-mail: popluciangh@icloud.com \\ DOI \\ 10.25122/jml-2021-0001 \\ Dates \\ Received: 1 January 2021 \\ Accepted: 19 February 2021
}

\begin{abstract}
Tuberculosis (TB) in pregnancy is not only a matter of the past; it is also a current problem. These days, TB appears through mass migration and tourism in countries where it was believed that this condition is eradicated. Adequate knowledge about the medical history of patients, risk factors, diagnosis and treatment of tuberculosis should be part of the armamentarium of each physician involved in clinical practice. TB is mainly found in urban and socially deprived areas. Due to the length of the treatment, there is an increased risk of drug resistance in partially treated patients. Strong knowledge about the history, risk factors, diagnosis and treatment of TB should be part of the armamentarium of each physician. Many practitioners are reluctant to request a chest X-ray in pregnancy due to the fear of harming the fetus. Bypassing a diagnosis can have a devastating effect on the mother and fetus, as well as their family and medical staff. This article discusses the matters of diagnosis and treatment of asymptomatic infection and active TB in pregnancy.
\end{abstract}

KEYWORDS: tuberculosis, infection, congenital.

\section{INTRODUCTION}

According to the World Health Organisation (WHO), tuberculosis (TB) is one of the most common causes of death worldwide, with approximately 1.5 million people dying every year. In 2018, there were 10 million people infected with TB, of which 3.2 million were women, many of them of reproductive age [1]. The incidence of TB on pregnancy reflects TB incidence overall, and there is a huge gap between developed and underdeveloped countries despite a $\%$ decrease per year [1]. A wide variation exists even among high-income countries, which is related to ethnic minorities, such as differences between white, non-Hispanic $(0.2 / 100000)$ and. Native Hawaiian/Pacific Islander (11.8/100000) population in the United States of America (USA) regarding women of fertile age [2]. Recent mass migration caused a TB relapse even in countries that rarely experienced TB cases, such as Sweden [3]. In the European Union, TB remains one of the significant health problems, with over 55537 cases reported in 2017. Romania has one of the highest incidences of TB, with 13004 cases reported [4]. Strong attention should be given to HIV-infected women, where TB incidence is ten times higher compared to HIV-negative women [5].

\section{Natural history}

Looking back at the natural history of tuberculosis, the disease was discovered by Robert Koch in 1892, and its name means fungus (Gk. mykes) and bakterion (Gk. little rod) and derives from the Greek language [6]. 


\section{JOURNAL of MEDICINE and LIFE}

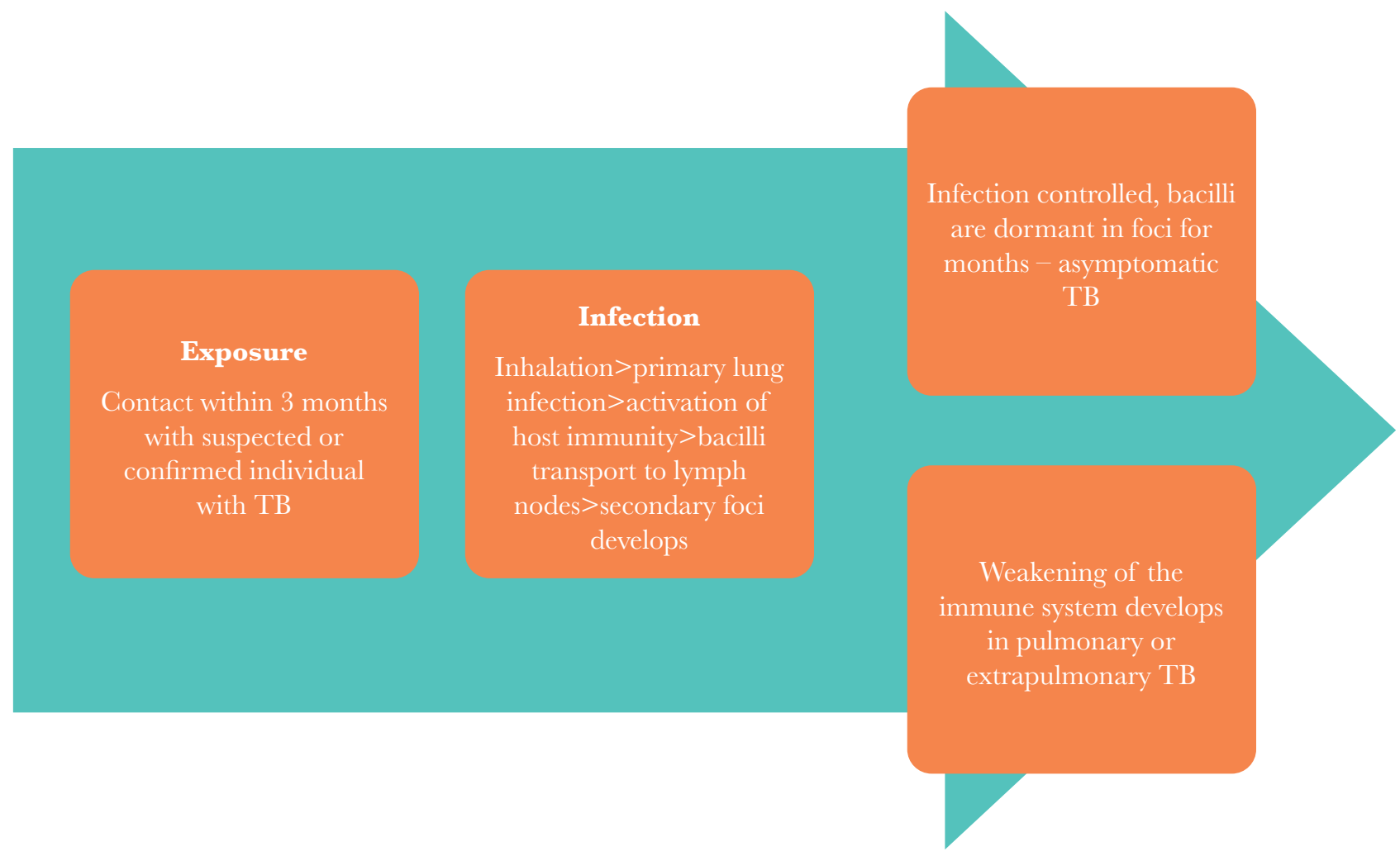

Figure 1. History of tuberculosis infection.

Mycobacterium tuberculosis is the most common bacteria encompassing $99 \%$ of the positive culture cases, while M. boviis and M. Africanum are accountable for only 1\%. There are several other types, such as Mycobacterium kansaii, Mycobacterium xenopi, Mycobacterium avium, and Mycobacterium fortuitum, that rarely cause disease in humans but do give positive results on the Mantoux tuberculin skin test and can cause resistance in HIV and TB patients [7]. Figure 1 shows the natural history of TB in an infected individual.

\section{Risk factors for TB in pregnancy}

The healthcare practitioner should keep in mind risk factors for TB, as screening for this condition could expedite the diagnosis. Screening in pregnancy is recommended by the World Health Organization (WHO) if the prevalence is higher than 100/100000 cases. This can be achieved in two ways: X-ray with protective shield and screening for symptoms compatible with TB [6], as shown in Table 1.

Table 1. Screening in pregnancy.

\begin{tabular}{|l|c|}
\hline Alvarez-Leon [8] & Family member with infectious cases \\
\hline Starke, JR [6] & $\begin{array}{r}\text { Travel to countries where TB is common, i.e., } \\
\text { developing Asian and African countries }\end{array}$ \\
\hline Janssens et al. [9] & $\begin{array}{r}\text { Living in ethnic minority communities where } \\
\text { TB is common }\end{array}$ \\
\hline Gupta A [10] & $\begin{array}{r}\text { Having an immune system damaged by HIV } \\
\text { or other health problems }\end{array}$ \\
\hline Mahendru A [11] & $\begin{array}{r}\text { Being extremely young or elderly, as the } \\
\text { immune system is less robust at these ages }\end{array}$ \\
\hline Lewlyn [12] & $\begin{array}{r}\text { Chronic poor health and nutrition because of } \\
\text { the standard of living; }\end{array}$ \\
Living in deprived or packed houses
\end{tabular}

Clinical diagnosis can only be made as long as there is an active disease and features are similar to the non-pregnant state and cough $(71 \%)$, weight loss $(41 \%)$, malaise, fatigue $(30 \%)$, fever $(30 \%)$ are the most common [6]. The lungs are the most common TB site, followed by the lymph nodes, bone, kidney, gastrointestinal system, and very rarely genital area, including the breast [7]. The typical location of $\mathrm{TB}$ can be modified in HIV-positive women, with unusual locations such as and the meninges, mediastinum, and kidneys; also, tuberculosis is more serious in these sites [11].

\section{The effect of pregnancy on tuberculosis}

It does not seem that pregnancy might have a harmful effect on tuberculosis as long as this condition is treated properly. 


\section{JOURNAL of MEDICINE and LIFE}

The TB prognosis is influenced by the extension of the condition, the radiographic model, and women's susceptibility to the disease. Pregnancy can indirectly influence patients with tuberculosis since many of the TB symptoms overlap with typical pregnancy signs such as fatigue, tachypnea, which can delay treatment and diagnosis. However, approximately one-half and two-thirds of the patients are in the latent stage, making diagnosis even more difficult [7].

\section{Table 2. Congenital tuberculosis - diagnostic criteria.}

Tuberculosis infection of the placenta or maternal genital tract

Hepatic primary complex or hepatic caseating granulomas

Lesions in the first week of life

Exclusion of postnatal transmission through investigation of contacts and strong guidelines compliance

\section{The effect of tuberculosis on pregnancy}

From reproduction to delivery and beyond, TB can influence all stages of the birth process. Infertility in the form of uterine synechia, tubal obstruction, implantation, and ovarian failure can all be consequences of TB. Even when pregnancy occurs, the risk of a tubal or abdominal pregnancy is higher than in unaffected women [13]. Prematurity and low birth weight risk increases by $2-3$ times and perinatal death in babies from a mother with tuberculosis increases by 6 times $[12,14]$.

So far, there are no data regarding the true incidence of TB infection in pregnancy; estimations show around 500,000 children aged 0-4 are infected with TB. Congenital transmission can occur via the amniotic fluid, by hematogenous spread, or both. Once the placenta is infected, the fetus can acquire the infection through the umbilical cord following a primary site in the liver with subsequent hematological dissemination or via amniotic fluid when the primary complex is within the lungs or gastrointestinal tract $[15,16]$. Newborn acquired infection must be distinguished from congenital infection. Aerosolized transmission after birth will result in pulmonary disease and is not considered congenital TB. Cantwell et al. developed easy-to-apply diagnostic criteria, which are well-suited with modern practice (Table 2) [17].

\section{Congenital tuberculosis}

Congenital tuberculosis occurs more frequently during pregnancy or delivery from primary maternal infection. In a study conducted by Peng et al., 121 mothers out of 162 had no previous history of tuberculosis infection, which is consistent with the available literature data. Maternal diagnosis of tuberculosis is difficult, with the majority of women being diagnosed postpartum and after their children were diagnosed with congenital tuberculosis. Tuberculosis can affect mothers' wellbeing, as it was showed by a Norwegian study, in terms of preeclampsia, postpartum hemorrhage, and difficult labor [18]. Extrapulmonary TB does not influence the pregnancy but can cause recurrent admission and higher maternal mortality rates when the central nervous system is affected [19].

In newborns, tuberculosis symptoms such as fever, respiratory distress, hepatosplenomegaly, cough, poor feeding, failure to thrive, cyanopathy, abdominal distension are non-specific and overlapping with bacterial sepsis, or congenital viral infections, which makes the diagnosis even more challenging. Clinical signs do not improve after antibiotics and their condition can deteriorate. Laboratory findings are common in multiple diseases, and for that reason, we underline Cantwell's criteria regarding caseating hepatic granulomas and TB infection in the placenta [20].

A fast and robust diagnosis can be achieved these days using imaging techniques such as chest X-ray, ultrasound, computed tomography (CT), and magnetic resonance imaging (MRI) with imaging-guided sampling, allowing early treatment.

\section{Table 3. Laboratory diagnosis.}

Investigation

Mantoux test $-0.1 \mathrm{ml}$ tuberculin skin test

\section{Interpretation}

$0-4 \mathrm{~mm}$ - no reaction

5-10 $\mathrm{mm}$ - doubtfully positive;

$10-15 \mathrm{~mm}$ - reactive in high risk cases;

$>15 \mathrm{~mm}$ - positive in all cases

Nodular shadow in the upper zone; Loss of volume, fibrosis, cavitation; Primary focus in latent TB
Depends on the site, useful for extrapulmonary TB

Gram-positive, acid-fast bacilli in culture 


\section{JOURNAL of MEDICINE and LIFE}

\section{Tuberculosis diagnosis in pregnancy}

The test of choice in TB diagnosis is the tuberculin skin test (Mantoux test). Lately, the interferon-gamma release assay (T-spot) test that measures interferon-gamma is used as well. Pregnant women have their immune function suppressed, but this does not appear to influence the test result compared with the immunosuppressed patient due to various conditions such as (HIV, drug addiction) where $40-60 \%$ of the test results are false-negative. When there is a clinical suspicion of TB, a thorough pursuit should follow, involving a chest X-ray with shield even below 12 weeks of gestation if the patient's condition is highly suggestive of TB (Table 3) [20-22].

\section{Treatment and Prognosis}

\section{Preventive therapy}

Isoniazid, which has no side effect on the fetus, is an effective treatment to prevent latent infection and active disease in susceptible individuals. The main shortcoming of isoniazid is its hepatic toxicity. The incidence of death due to isoniazid is $1 / 20,000$. The American Thoracic Society recommends that for most women, preventive treatment should be delayed after delivery with the notable exception of an HIV-positive individual or contact with a contagious person. Preventive treatment should be delayed after the first trimester [21-24].

\section{Management of drug sensitive-TB in pregnancy}

Currently, TB therapeutic schemes are limited in providing evidence-based advice as pregnant women are usually excluded from trials due to fears of teratogenicity.

Pregnant women with pulmonary drug-sensitive-TB may be treated with either the standard four-drug scheme or a three-drug scheme without pyrazinamide and prolonging the treatment for 9 months. According to WHO, there is no specific treatment for TB in pregnancy but using pyrazinamide in pregnancy is under question in some countries due to the lack of safety data during gestation [25]. There are particular conditions when the benefits of using pyrazinamide outweigh the risks, such as in HIV/TB patients or those with extrapulmonary disease. Also, streptomycin should not be used in pregnancy as it causes ototoxicity. Vitamin K should be prescribed to the fetus at birth to reduce the risk of hemolytic anemia due to rifampicin.

\section{Management of drug resistant-TB in pregnancy}

The most challenging issue regarding TB treatment is drug-resistant tuberculosis. Second-line drugs may have a harmful effect on the fetus. Ethionamide, cycloserine are category C drugs that have showed teratogenic effects in animals. Aminoglycosides such as the aforementioned streptomycin or kanamycin can cause ototoxicity [26]. The fluoroquinolones are harming the growing cartilage and should be avoided entirely in pregnancy if possible. In drug-resistant TB, the treatment is usually prolonged to 24 months [24].

A complex issue is the management of HIV-positive patients during pregnancy, particularly for that category of patients not yet receiving antiretroviral therapy (ART). Multidisciplinary team management, according to the recent guidelines, is mandatory.

\section{CONCLUSION}

Usually, tuberculosis is seen in endemic areas; nevertheless, the consequences of a missed diagnosis could be tragic, increasing the risk of miscarriage, perinatal death, or maternal death. TB should be suspected in any woman coming from an endemic area with non-specific symptoms and clinical signs. Also, pregnancy makes this condition even more challenging both in terms of diagnosis and treatment with limited guidance and data regarding second-line drugs in pregnancy. The present barrier in screening and diagnosis represents a risk for both mothers and healthcare professionals. Whether preventive therapy should be prescribed during the pregnancy or suspended until delivery still requires an answer. For this reason, future research should focus on drug safety, prevention therapy, and optimizing screening programs.

\section{ACKNOWLEDGEMENTS}

\section{Conflict of interest}

The authors declare that there is no conflict of interest. 


\section{JOURNAL of MEDICINE and LIFE}

\section{REFERENCES}

1. https://www.who.int/news-room/fact-sheets/detail/ tuberculosis. Accesed on 30.12.2020

2. Gould JM, Aronoff SC. Tuberculosis and Pregnancy-Maternal, Fetal, and Neonatal Considerations. Microbiol Spectr.

2016;4(6):10.1128/microbiolspec.TNMI7-0016-2016. doi:10.1128/microbiolspec.TNMI7-0016-2016.

3. Borisov S, Danila E, Maryandyshev A, et al. Surveillance of adverse events in the treatment of drug-resistant tuberculosis: first global report. Eur Respir J. 2019:54(6): 1901522. Published 2019 Dec 19. doi:10.1183/13993003.01522-2019

4. https://www.ecdc.europa.eu/sites/default/files/ documents/TB-Surveillance-report_24March2020. Accessed on 28.12 .2021

5. Adhikari M. Tuberculosis and tuberculosis/HIV co-infection in pregnancy. Semin Fetal Neonatal Med. 2009;14(4):234-240. doi:10.1016/j.siny.2009.02.001.

6. Starke JR. Tuberculosis. An old disease but a new threat to the mother, fetus, and neonate. Clin Perinatol. 1997;24(1):107-127

7. Wilson, EA, Thelin TJ, and Dilts PV. Tuberculosis complicated by pregnancy. Am J Obstet Gynecol, 1973. 115(4): p. 526-9.

https://doi.org/10.1016/0002-9378(73)90401-8.

8. Alvarez-León EE, Espinosa-Vega E, Santana-Rodríguez E, et al. Screening for tuberculosis infection in spanish healthcare workers: Comparison of the QuantiFERON-TB gold in-tube test with the tuberculin skin test. Infect Control Hosp Epidemiol. 2009;30(9):876-883. doi:10.1086/598519.

9. Janssens, JP. Clinical utility of the interferon-\{gamma\} release assay for elderly patients with active tuberculosis: a word of caution. Chest, 2008. 134(2): p. 471-472.

10. Gupta A, Montepiedra G, Theron G. HIV, Pregnancy, and Isoniazid Preventive Therapy. Reply. N Engl J Med. 2020;382(12):1184-1185. doi:10.1056/NEJMc1916664.
11. Mahendru A, Gajjar K, Eddy J. Diagnosis and management of tuberculosis in pregnancy. 2010. 12:163-171. https://doi.org/10.1576/toag. 12.3.163.27598

12. Llewelyn M, Cropley I, Wilkinson RJ, Davidson RN. Tuberculosis diagnosed during pregnancy: a prospective study from London. Thorax. 2000;55(2):129-132. doi:10.1136/ thorax.55.2.129.

13. Muir DG, Belsey MA. Pelvic inflammatory disease and its consequences in the developing world. Am J Obstet Gynecol. 1980;138(7 Pt 2):913-928.

doi:10.1016/0002-9378/80)91082-0.

14. Jana N, Vasishta K, Jindal SK, Khunnu B, Ghosh K. Perinatal outcome in pregnancies complicated by pulmonary tuberculosis. Int J Gynaecol Obstet. 1994;44(2):119-124. doi:10.1016/0020-7292(94)90064-7.

15. Hageman J, Shulman S, Schreiber M, Luck S, Yogev R. Congenital tuberculosis: critical reappraisal of clinical findings and diagnostic procedures. Pediatrics. 1980;66(6):980-984.

16. Hamadeh MA, Glassroth J. Tuberculosis and pregnancy. Chest. 1992;101(4):1114-1120. doi:10.1378/chest.101.4.1114.

17. Cantwell MF, Shehab ZM, Costello AM, et al. Brief report: congenital tuberculosis. N Engl J Med. 1994;330(15): 1051-1054.

doi:10.1056/NEJM199404143301505

18. Bjerkedal T, Bahna SL, Lehmann EH. Course and outcome of pregnancy in women with pulmonary tuberculosis. Scand J Respir Dis. 1975;56(5):245-250.

19. Jana N, Vasishta K, Saha SC, Ghosh K. Obstetrical outcomes among women with extrapulmonary tuberculosis. N Engl J Med. 1999;341(9):645-649. doi:10.1056/NEJM199908263410903.
20. Peng W, Yang J, Liu E. Analysis of 170 cases of congenital TB reported in the literature between 1946 and 2009. Pediatr Pulmonol. 2011;46(12):1215-1224. doi:10.1002/ppul.21490.

21. Laurenti P, Raponi M, de Waure C, Marino M Ricciardi W, Damiani G. Performance of interferon- $\gamma$ release assays in the diagnosis of confirmed active tuberculosis in immunocompetent children: a new systematic review and meta-analysis. BMC Infect Dis. 2016;16:131. Published 2016 Mar 18. doi:10.1186/s12879-016-1461-y.

22. WHO, Recommendations on antenatal care for a positive pregnancy experience 2016

23. https://www.cdc.gov/tb/publications/ltbi. Accessed on 29.12.2020

24. American Thoracic Society; CDC; Infectious Diseases Society of America. Treatment of tuberculosis [published correction appears in MMWR Recomm Rep. 2005 Jan 7;53(51):1203. Dosage error in article text]. MMWR Recomm Rep. 2003;52(RR-11):1-77.

25. Nahid P, Dorman SE, Alipanah N, et al. Official American Thoracic Society/Centers for Disease Control and Prevention/Infectious Diseases Society of America Clinical Practice Guidelines: Treatment of Drug-Susceptible Tuberculosis. Clin Infect Dis. 2016;63(7):e147-e195. doi:10.1093/cid/ciw376.

26. Nhan-Chang CL, Jones TB. Tuberculosis in pregnancy. Clin Obstet Gynecol. 2010;53(2):311-321. doi:10.1097/GRF.0b013e3181de8a13. 